\title{
Theracurmin (Highly Bioavailable Curcumin) Prevents High Fat Diet-Induced Hepatic Steatosis Development in Mice
}

\author{
Jin Won Yang ${ }^{1}$, Hee Kyung Yeo ${ }^{2}$, Jee Hye Yun² and Jung Un Lee \\ ${ }^{1}$ College of Pharmacy, Woosuk University, Wanju, Korea \\ ${ }^{2}$ HANDOK Inc., Seoul, Korea \\ ${ }^{3}$ ChemOn Inc., Suwon, Korea
}

\begin{abstract}
Curcumin, a hydrophobic polyphenol isolated from the Curcuma longa L. plant, has many pharmacological properties, including antioxidant, anti-inflammatory, and chemo-preventive activities. Curcumin has been shown to have potential in preventing nonalcoholic fatty liver disease (NAFLD). However, the low bioavailability of curcumin has proven to be a major limiting factor in its clinical adoption. Theracurmin, a highly bioavailable curcumin that utilizes micronized technology showed improved biological absorbability in vivo. The aim of this study was to investigate the role of theracurmin in modulating hepatic lipid metabolism in vivo. A fatty liver mouse model was produced by feeding mice a high fat diet (HFD; 60\% fat) for 12 weeks. We found that treatment for 12 weeks with theracurmin significantly lowered plasma triacylglycerol (TG) levels and reduced HFD-induced liver fat accumulation. Theracurmin treatment lowered hepatic TG and total cholesterol (T-CHO) levels in HFD-fed mice compared to controls. In addition, theracurmin administration significantly reduced lipid peroxidation and cellular damage caused by reactive oxygen species in HFD-fed mice. Overall, these results suggest that theracurmin has the ability to control lipid metabolism and can potentially serve as an effective therapeutic remedy for the prevention of fatty liver.
\end{abstract}

Key words: Theracurmin, Curcumin, Nonalcoholic fatty liver disease (NAFLD), High fat diet (HFD), Fatty liver, Steatosis

\section{INTRODUCTION}

Non-alcoholic fatty liver disease (NAFLD) has been recognized as a common liver disease worldwide. The term represents a broad spectrum of liver damage ranging from simple steatosis to nonalcoholic steatohepatitis (NASH), progressive fibrosis, and cirrhosis $(1,2)$. NAFLD is strongly associated with metabolic syndrome-associated conditions such as obesity, dyslipidemia, diabetes, hypertension, and insulin resistance $(3,4)$. The detailed pathogenesis of

Correspondence to: Jin Won Yang, College of Pharmacy, Woosuk University, 443 Samnyero, Samnye-eup, Wanju-gun, Jeonbuk 55338, Korea

E-mail: jwyang@woosuk.ac.kr

This is an Open-Access article distributed under the terms of the Creative Commons Attribution Non-Commercial License (http:// creativecommons.org/licenses/by-nc/3.0) which permits unrestricted non-commercial use, distribution, and reproduction in any medium, provided the original work is properly cited.
NAFLD is not completely known, but excessive fatty acid [triacylglycerol (TG)] and cholesterol (CHO) accumulation in the liver has been linked to the development of NASH, cirrhosis, and cancer $(5,6)$. Although current therapeutic approaches have focused on the treatment of the underlying risk factors for these metabolic conditions, no standard strategy has yet been approved for NAFLD therapy $(2,6)$.

Curcumin, a natural yellow polyphenol that exists in herbal remedies and the dietary spice turmeric, has been
List of Abbreviations: ACAT, acyl-CoA:cholesterol acyltransferase; GSH, glutathione; $\mathrm{HDL}$, high-density lipoprotein; $\mathrm{H} \& \mathrm{E}$, hematoxylin and eosin; HFD, high fat diet; HMG-CoA, 3-hydroxy-3methylglutaryl-CoA; LDL, low-density lipoprotein; MDA, malondialdehyde; NAFLD, Nonalcoholic fatty liver disease; NASH, nonalcoholic steatohepatitis; ND, normal diet; Nrf2, nuclear factor erythroid-2-related factor-2; ROS, reactive oxygen species; SREBPs, sterol regulatory elementbinding proteins; TBA, thiobarbituric acid; T-CHO, Total cholesterol; TG, triacylglycerol. 
shown to possess antioxidant, anti-inflammatory, antimicrobial, and chemopreventive activities, and has been demonstrated to prevent obesity and diabetes in animal models $(7,8)$. Curcumin also exerted beneficial effects against hypercholesterolemia and dyslipidemia in rodent animal models, as well as in two randomized double-blind NAFLD clinical trials $(9,10)$.

Although curcumin has been shown to be protective against dyslipidemia and NAFLD, its therapeutic outcomes and clinical use is limited by low oral bioavailability owing to its very low intestinal absorption and hydrophobic properties, leading to poor solubility (11-13). Many studies investigating curcumin delivery systems, including submicron suspensions, phosphatidylcholine complexes, and solid lipid nanoparticles, have been performed with the aim of improving curcumin oral bioavailability (1416). Among these, thearcurmin, a highly bioavailable curcumin developed using micronized-technology, has significantly increased bioavailability and water solubility relative to curcumin. In rat and human studies, theracurmin absorption was 30 -fold higher than that of commercially available curcumin (14). Moreover, the maximum curcumin plasma concentration increased over 50-fold when theracurmin was used instead of curcumin powder (14).

Theracurmin has been reported to be effective against a variety of pathological conditions including cardiovascular disease, esophageal cancer, inflammatory bowel disease, and osteoarthritis $(11,14,17)$. However, no basic or clinical studies regarding the efficacy of theracurmin against NAFLD, including hepatic steatosis, have been performed. In this study, we evaluated the preventive effect of theracurmin on hepatic steatosis in mice fed with a high fat diet (HFD). We uncovered that theracurmin treatment prevented the accumulation of TG and total cholesterol (T$\mathrm{CHO}$ ), as well as the lipid peroxidation, normally observed in the livers of HFD-fed mice. Our findings suggested that theracurmin is protective against NAFLD through lipid metabolism and oxidative stress modulation, and has therapeutic potential.

\section{MATERIALS AND METHODS}

Animals and treatment. Animal experiments were performed in accordance with the requirements of the Animal Care and Ethics Committees of Gyeonggi bio center (2017-08-0001). C57BL/6N mice at 4 weeks of age were maintained in a standard condition $\left(23 \pm 3^{\circ} \mathrm{C}, 55 \pm\right.$ $15 \%$ humidity with a 12 -hr light/dark cycle), pathogenfree environment and had access to a sterile standard rodent chow diet and water ad libitum. After a one week adaptive period, Male C57BL/6N mice at 5 weeks of age were started on either a normal diet (ND) or HFD $60 \% \mathrm{w} / \mathrm{w}$ for 12 weeks. Vehicle (normal saline), theracurmin (500, 1,000 , and $2,000 \mathrm{mg} / \mathrm{kg}$, as a curcumin $150,300,600 \mathrm{mg} /$ $\mathrm{kg})$, or silymarin $(25 \mathrm{mg} / \mathrm{kg})$ were orally administered to mice seven times per week during 12 weeks of the diet feeding.

Histopathological analysis. The left lateral lobe of the liver was sliced and tissue slices were fixed in $10 \%$ buffered-neutral formalin, embedded in paraffin. The liver slices were used to generate 3-4 $\mu \mathrm{m}$ sections in a cryostat. Tissue sections were stained with $\mathrm{H} \& \mathrm{E}$ and Oil red $\mathrm{O}$ staining.

After that the histological profiles of individual cross trimmed hepatic tissues were light microscopically observed (Model Eclipse 80i, Nikon, Tokyo, Japan). To observe more detail histopathological changes, the steatohepatitis regions (under OR staining) and mean hepatocyte diameters (under HE staining) were calculated using an automated image analysis process (iSolution FL ver 9.1, IMT $i$-solution Inc., Vancouver, Quebec, Canada) on the restricted view fields. Steatohepatitis regions, the percentage of fatty deposited regions in hepatic parenchyma, were calculated as percentages of lipid deposited regions between restricted histological view field of liver (Mean hepatic steatosis regions $-\% / \mathrm{mm}^{2}$ of hepatic parenchyma) under cryostat and oil red staining. Mean diameters of hepatocytes were also calculated in restricted view fields on a computer monitor under paraffin embedding and HE staining using an automated image analysis process.

Measurement of hepatic TG and total cholesterol contents. TG (Catalog\#K622-100, Biovision, San Francisco, CA, USA) and T-CHO (Catalog\#K603, Biovision) contents were measured using commercial kits.

Biochemical parameters. Serum was collected after centrifugation at 3,000 rpm for $10 \mathrm{~min}$. Serum TG (Catalog\#OSR61118, Beckman coulter, CA, USA), T-CHO (Catalog\#OSR6116, Beckman coulter), LDL-C (Catalog\#6183, Beckman coulter), and HDL-C (Catalog\#OSR6187, Beckman coulter), were analyzed using commercial kits from Chemistry Analyzer (AU680, Beckman coulter).

Measurement of hepatic malondialdehyde and glutathione contents. Malondialdehyde (MDA) levels was determined by the thiobarbituric acid (TBA) method (Catalog\#STA-330, Cellbiolabs, San Francisco, CA, USA). TBA reaction was performed according to manufactory guidance. Glutathione (GSH) were analyzed using commercial kits (Catalog\#ADI-900-160, ENZO Life Science, Vileurbanne, France).

Data analysis. Statistical analyses were performed using SPSS statistics 22 for medical science. LSD test was used to examine the significant inter-group differences. Statistical significance was accepted at either $p<0.05$ or $p<0.01$. 


\section{RESULTS}

Theracurmin inhibits HFD-induced hepatic steatosis.

To examine the effect of theracurmin on liver fat accumu- lation, mice were fed with a HFD (60\% fat) for 12 weeks and then treated with vehicle, theracurmin, or silymarin $(25$ $\mathrm{mg} / \mathrm{kg}$; reference control). Since the main histological feature of hepatic steatosis is liver fat accumulation, hepatic

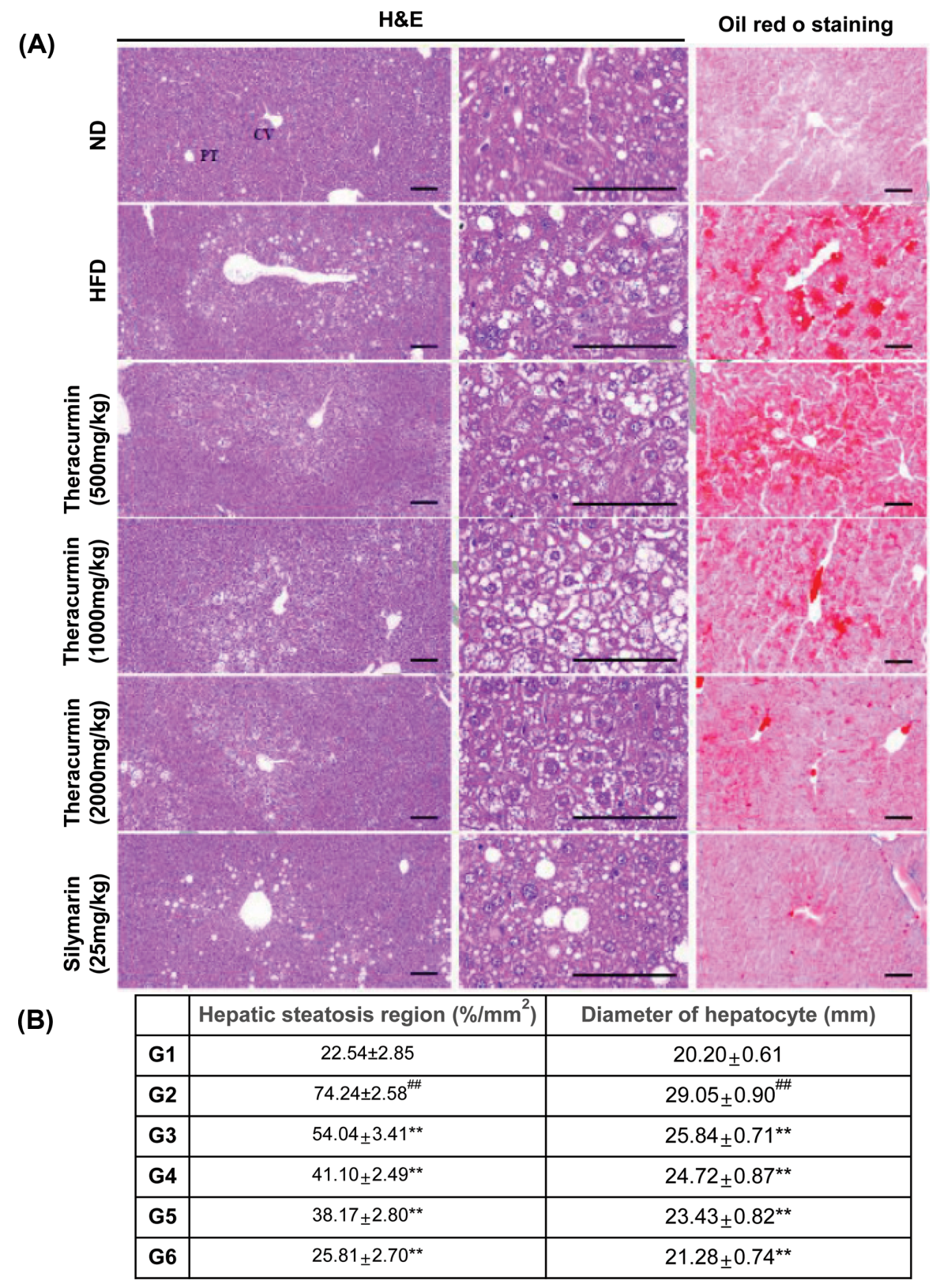

Fig. 1. Effects of theracurmin on hepatic lipid accumulation in mice fed with HFD. (A) Liver sections were stained with H\&E or oilRed O staining. The mice were fed either a ND or HFD for 12 weeks. theracurmin $(500,1,000$, and 2,000 mg/kg) or silymarin ( $25 \mathrm{mg} /$ $\mathrm{kg}$ ) (reference control) was administered to the mice at the same time. H\&E staining. The livers of the mice were stained with H\&E after a treatment with 500, 1,000, and 2,000 mg/kg theracurmin for 12 weeks. CV, Central vein; PT, Portal triad area; Scale bars = $100 \mu \mathrm{m}$. Oil Red O staining. Each photo represents their groups after staining with Oil Red $\mathrm{O}$ in the liver. Scale bars $=100 \mu \mathrm{m}$. (B) Histomorphometric analysis. Measurement of hepatic steatosis region $\left(\% / \mathrm{mm}^{2}\right.$ of hepatic parenchyma) and diameter of hepatocyte $(\mathrm{mm} /$ hepatocyte). Data were expressed as mean \pm SEM statistically analyzed by LSD-test methods. Significant versus normal control, ${ }^{\# \#} p<0.01$; significant versus HFD-fed group, ${ }^{* *} p<0.01 \quad(\mathrm{n}=10)$. G1: ND (normal saline), $\mathrm{n}=10 ; \mathrm{G} 2$ : HFD + vehicle (normal saline), $\mathrm{n}=10 ; \mathrm{G} 3$ : HFD + theracurmin (500 mg/kg/day), $\mathrm{n}=10 ; \mathrm{G} 4$ : HFD + theracurmin $(1,000 \mathrm{mg} / \mathrm{kg} /$ day $), \mathrm{n}=10 ; \mathrm{G} 5$ : HFD + theracurmin (2,000 mg/kg/day), $\mathrm{n}=10 ; \mathrm{G} 6:$ HFD + reference control (silymarin $25 \mathrm{mg} / \mathrm{kg} / \mathrm{day}), \mathrm{n}=10$. 

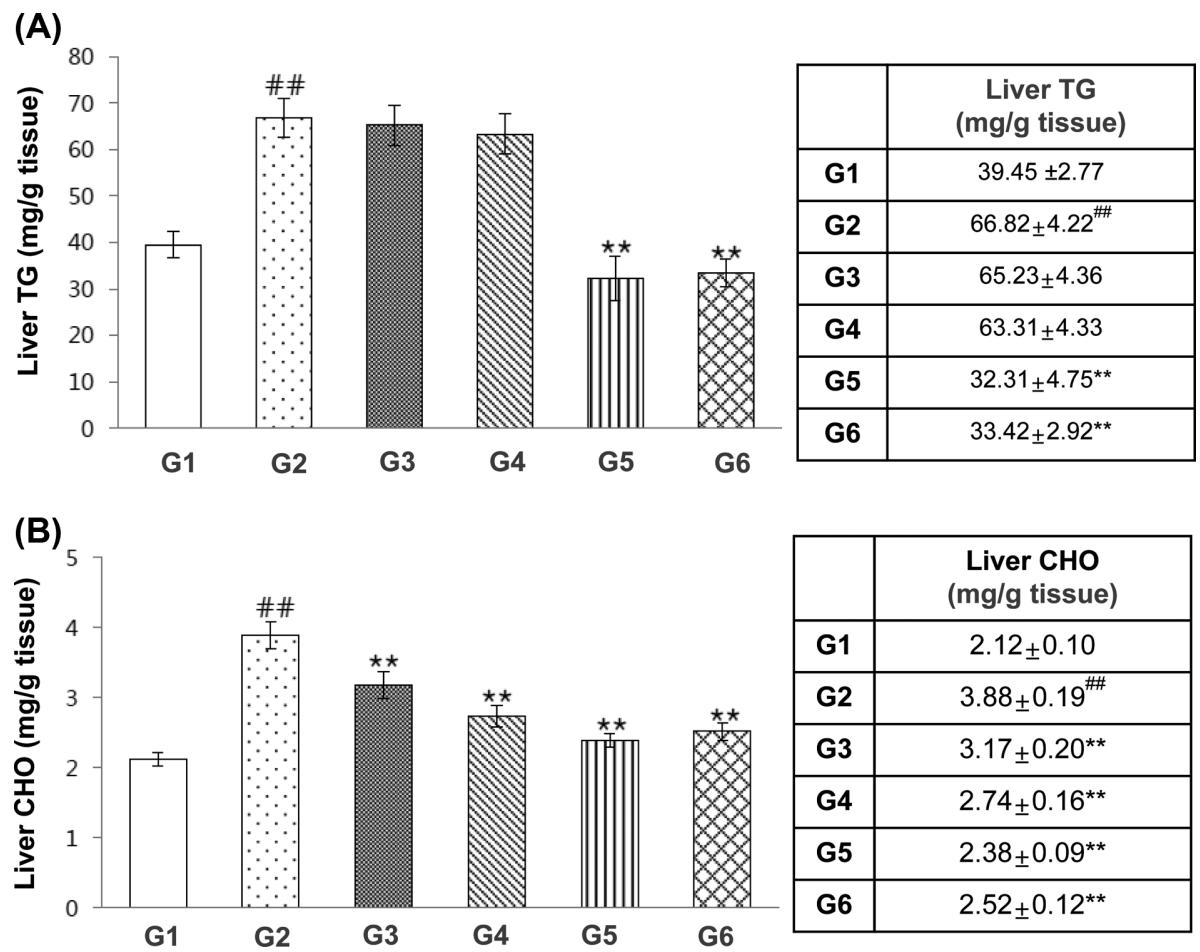

Fig. 2. Effects of theracurmin on the accumulation of hepatic $T G$ and $C H O$ in HFD-fed mice. $(A, B)$ Measurement of accumulation of TG and $\mathrm{CHO}$ in the liver from mice of each group. Data were expressed as mean \pm SEM statistically analyzed by LSD-test methods. Significant versus normal control, ${ }^{\#} p<0.01$; significant versus HFD-fed group, ${ }^{* *} p<0.01(\mathrm{n}=10)$. G1: ND (normal saline), $\mathrm{n}=10$; G2: HFD + vehicle (normal saline), $\mathrm{n}=10 ; \mathrm{G} 3$ : HFD + theracurmin $(500 \mathrm{mg} / \mathrm{kg} / \mathrm{day}), \mathrm{n}=10 ; \mathrm{G} 4: \mathrm{HFD}+$ theracurmin $(1,000 \mathrm{mg} / \mathrm{kg} / \mathrm{day})$, $\mathrm{n}=10 ; \mathrm{G5}: \mathrm{HFD}+$ theracurmin (2,000 mg/kg/day), $\mathrm{n}=10 ; \mathrm{G6}$ : HFD + reference control (silymarin $25 \mathrm{mg} / \mathrm{kg} /$ day), $\mathrm{n}=10$.

fat deposition was measured. Histopathological analysis using hematoxylin and eosin (H\&E) and Oil Red O tissue staining found that HFD fed mice exhibited increased hepatocyte fat accumulation (Fig. 1A). Theracurmin treatment at doses of $500,1,000$, and $2,000 \mathrm{mg} / \mathrm{kg}$ significantly reduced these pathological changes in the tissue, as did oral administration of silymarin (Fig. 1B).

Theracurmin improves the accumulation hepatic TG and T-CHO in HFD-fed mice. TG and T-CHO accumulation in hepatocyte cytoplasm is the hallmark of NAFLD (1). Theracurmin treatment $(2,000 \mathrm{mg} / \mathrm{kg})$ for 12 weeks reduced HFD-induced hepatic TG content increases, as did oral administration of silymarin (Fig. 2A). In addition, hepatic $\mathrm{CHO}$ levels were significantly decreased in theracurmin-treated mice relative to ND controls [ND: $2.12 \pm 0.10 \mathrm{mg} / \mathrm{dL} ; \mathrm{HFD}, 3.88 \pm 0.19 \mathrm{mg} / \mathrm{dL} ; \mathrm{HFD}+$ Theracurmin $(500 \mathrm{mg} / \mathrm{kg}), 3.17 \pm 0.20 \mathrm{mg} / \mathrm{dL} ; \mathrm{HFD}+$ Theracurmin $(1,000 \mathrm{mg} / \mathrm{kg}), 2.74 \pm 0.16 \mathrm{mg} / \mathrm{dL} ; \mathrm{HFD}+$ Theracurmin $(2,000 \mathrm{mg} / \mathrm{kg}), 2.38 \pm 0.09 \mathrm{mg} / \mathrm{dL}$ ] (Fig. 2B). Accordingly, HFD treatment for 12 weeks induced increases in hepatocyte and hepatic steatosis region diameter increases, phenomena notably attenuated by administrations of theracurmin at doses of 500, 1,000, and 2,000 $\mathrm{mg} / \mathrm{kg}$ (Fig. 1B).
Theracurmin reduces plasma TG but does not affect body weight in HFD-fed mice. Next, we examined the effect of theracurmin on serum lipid levels. Theracurmin treatment significantly reduced HFD-induced plasma TG level increases but did not affect plasma total $\mathrm{CHO}$, lowdensity lipoprotein (LDL), and high-density lipoprotein (HDL) levels (Fig. 3). However, the administration of theracurmin did not affect body weight gain or food intake amounts (Supplementary Fig. 1).

Theracurmin inhibits HFD-induced lipid peroxidation. Lipid peroxidation and cellular damage by reactive oxygen species is characterized by membrane lipid breakdown and the production of lipid peroxides (18). As observed in Fig. 4A, liver MDA production, a marker of lipid peroxidation, was elevated in HFD-treated mice relative to normal diet-fed controls. This elevation of hepatic MDA was significantly attenuated by theracurmin administration at a dosage of $2,000 \mathrm{mg} / \mathrm{kg}$. Theracurmin treatment (1,000 and 2,000 $\mathrm{mg} / \mathrm{kg}$ doses) also increased hepatic GSH content versus untreated HFD-fed mice (Fig. 4B).

\section{DISCUSSION}

Curcumin has been shown to reduce hepatic steatosis, 
(A)

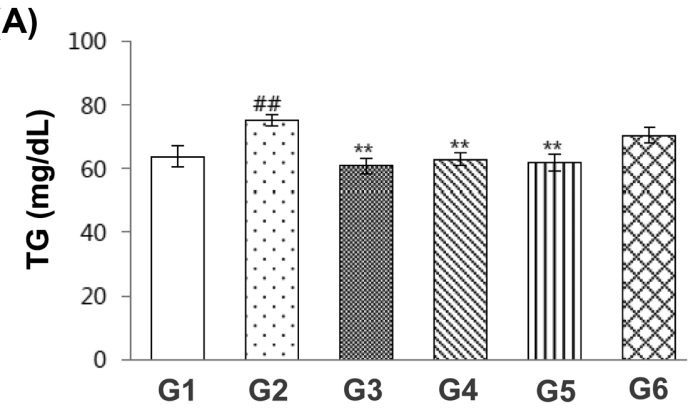

(B)

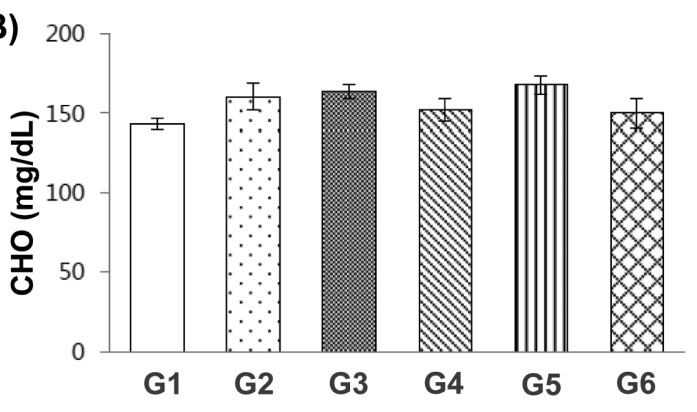

(C)

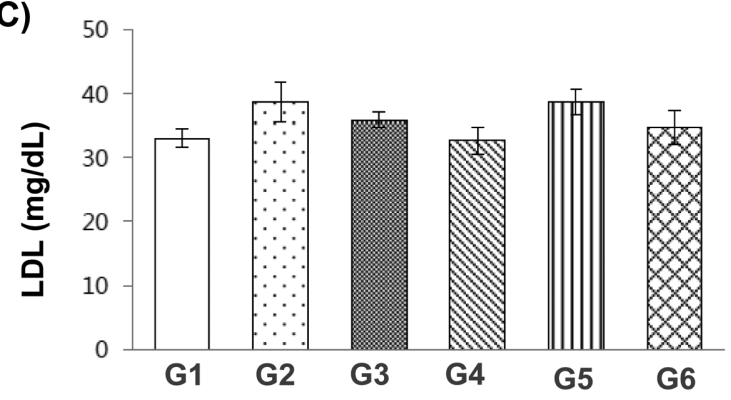

(D)

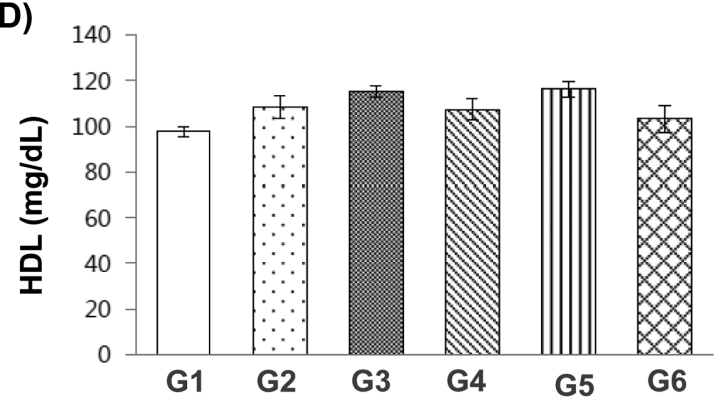

\begin{tabular}{|c|c|}
\hline & TG $(\mathrm{mg} / \mathrm{dL})$ \\
\hline G1 & $63.89 \pm 3.48$ \\
\hline G2 & $75.20 \pm 1.85^{\# \#}$ \\
\hline G3 & $60.90_{ \pm} 2.52^{\text {** }}$ \\
\hline G4 & $63.00_{ \pm} 2.07^{\text {** }}$ \\
\hline G5 & $61.78_{ \pm} 2.71^{\star \star}$ \\
\hline G6 & $70.50_{ \pm} 2.34$ \\
\hline
\end{tabular}

\begin{tabular}{|c|c|}
\hline & CHO $(\mathrm{mg} / \mathrm{dL})$ \\
\hline G1 & $143.00 \pm 3.48$ \\
\hline G2 & $160.30 \pm 8.13$ \\
\hline G3 & $163.50_{ \pm} 4.05$ \\
\hline G4 & $151.70_{ \pm} 7.14$ \\
\hline G5 & $167.89_{ \pm} 5.77$ \\
\hline G6 & $149.90_{ \pm} 9.38$ \\
\hline
\end{tabular}

\begin{tabular}{|c|c|}
\hline & LDL $(\mathrm{mg} / \mathrm{dL})$ \\
\hline G1 & $33.00 \pm 1.39$ \\
\hline G2 & $38.80 \pm 3.10$ \\
\hline G3 & $35.90_{ \pm} 1.27$ \\
\hline G4 & $32.70_{ \pm} 2.08$ \\
\hline G5 & $38.67 \pm 1.95$ \\
\hline G6 & $34.70 \pm 2.61$ \\
\hline
\end{tabular}

\begin{tabular}{|c|c|}
\hline & HDL $(\mathrm{mg} / \mathrm{dL})$ \\
\hline G1 & $97.58 \pm 2.21$ \\
\hline G2 & $108.28 \pm 4.80$ \\
\hline G3 & $115.14 \pm 2.42$ \\
\hline G4 & $107.32 \pm 4.68$ \\
\hline G5 & $116.26 \pm 3.48$ \\
\hline G6 & $103.18 \pm 5.87$ \\
\hline
\end{tabular}

Fig. 3. Effects of theracurmin on the serum TG, CHO, LDL, and $H D L$ in HFD-fed mice. (A-D) serum TG levels (A), serum CHO levels (B), serum LDL levels $(C)$, serum HDL levels $(D)$ in mice fed a normal diet or high-fat-diet for 12 weeks. Data were expressed as mean \pm SEM statistically analyzed by Q-test and LSD-test methods. Significant versus normal control, ${ }^{\# \# ~} p<0.01$; significant versus HFD-fed group, ${ }^{* *} p<0.01(\mathrm{n}=10$ ). G1: ND (normal saline), $\mathrm{n}=10 ; \mathrm{G} 2$ : HFD + vehicle (normal saline), $\mathrm{n}=10 ; \mathrm{G}$ : HFD + theracurmin (500 mg/kg/day), $\mathrm{n}=10 ; \mathrm{G} 4: \mathrm{HFD}+$ theracurmin $(1,000 \mathrm{mg} / \mathrm{kg} /$ day), $\mathrm{n}=10 ; \mathrm{G5}: \mathrm{HFD}+$ theracurmin $(2,000 \mathrm{mg} / \mathrm{kg} /$ day $), \mathrm{n}=10 ; \mathrm{G} 6$ : $\mathrm{HFD}+$ reference control (silymarin $25 \mathrm{mg} / \mathrm{kg} /$ day), $\mathrm{n}=10$.

inflammation, insulin resistance, diabetes, and atherosclerosis by regulating hepatic lipid metabolism and plasma lipid homeostasis $(19,20)$. However, the low oral bioavailability of curcumin limits its clinical adoption (11). To overcome this, theracumin, a submicron crystal solid dispersion of curcumin, was formulated to enhance cur- cumin bioavailability through enhanced water solubility and absorption $(7,11)$.

We demonstrate herein that theracurmin administration exerted anti-steatotic activity in the livers of mice fed with high fat diets. In the current study, theracurmin administration led to significant decreases in both hepatic TG and 

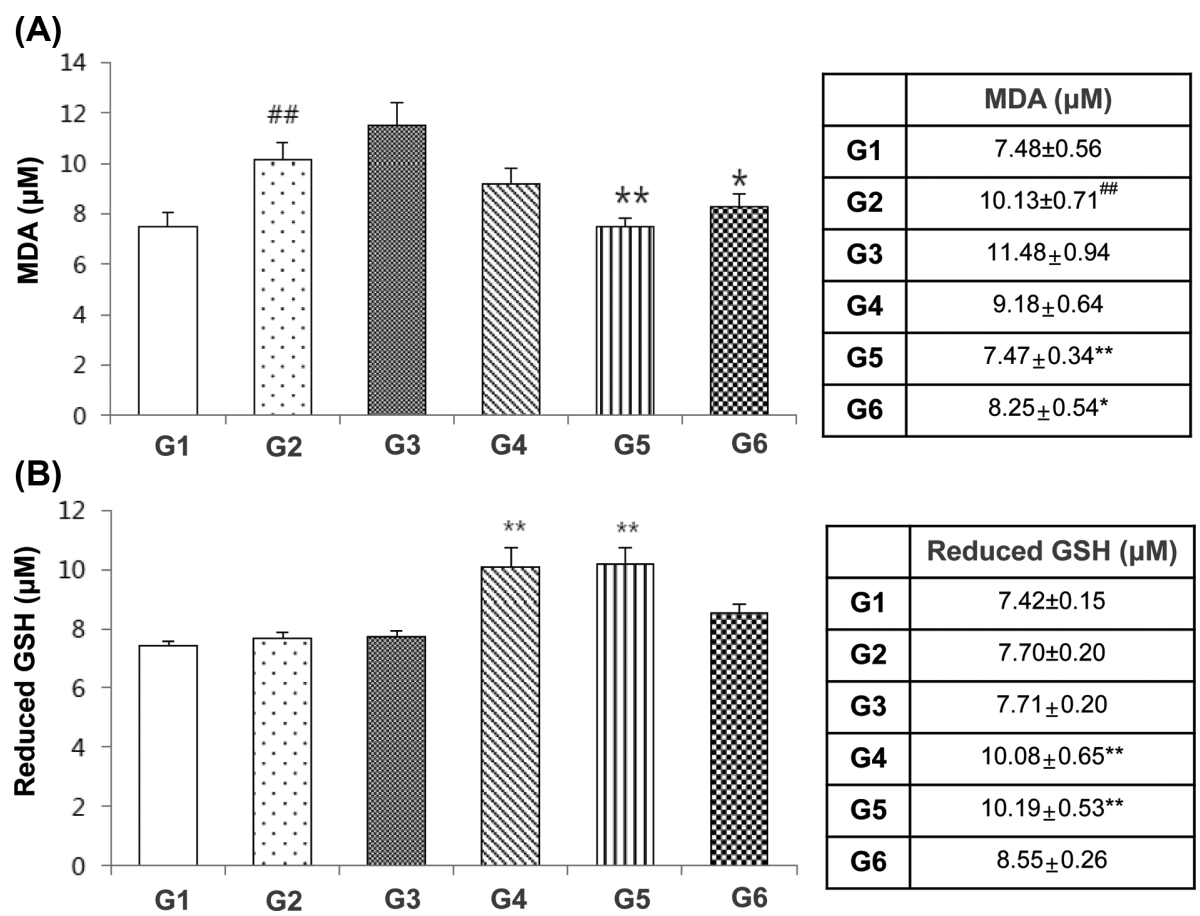

Fig. 4. Effects of theracurmin on oxidative stress in HFD-fed mice. Measurement of hepatic MDA and GSH contents in the liver from mice of each group. Data were expressed as mean \pm SEM statistically analyzed by Q-test and LSD-test methods. Significant versus normal control, ${ }^{\# \#} p<0.01$; significant versus HFD-fed group, ${ }^{*} p<0.05 ;{ }^{*} p<0.01$ ( $\mathrm{n}=10$ ). G1: ND (normal saline), $\mathrm{n}=10 ; \mathrm{G} 2$ : HFD + vehicle (normal saline), $\mathrm{n}=10 ; \mathrm{G} 3$ : HFD + theracurmin ( $500 \mathrm{mg} / \mathrm{kg} /$ day), $\mathrm{n}=10 ; \mathrm{G} 4: \mathrm{HFD}+$ theracurmin $(1,000 \mathrm{mg} / \mathrm{kg} / \mathrm{day})$, $\mathrm{n}=10 ; \mathrm{G5}: \mathrm{HFD}$ + theracurmin $(2,000 \mathrm{mg} / \mathrm{kg} /$ day), $\mathrm{n}=10 ; \mathrm{G} 6: \mathrm{HFD}$ + reference control (silymarin $25 \mathrm{mg} / \mathrm{kg} /$ day), $\mathrm{n}=10$.

total CHO levels in mice fed a HFD for 12 weeks. Excessive fatty acids, derived from diet or lipolysis, results in hepatocyte lipid droplet accumulation, a representative feature of NAFLD (21). Consequently, specific lipotoxic lipids, including ceramide, diacylglycerols, and lysophosphatidyl choline species, from these droplets induce hepatocellular injury in NASH $(2,21)$. In addition, an imbalance between intrahepatic $\mathrm{CHO}$ and the removal of $\mathrm{CHO}$ from hepatocytes leads to $\mathrm{CHO}$ accumulation in the liver $(22,23)$. This extensive dysregulation of hepatic $\mathrm{CHO}$ homeostasis has been shown to accentuate hepatocellular injury and liver inflammation in NAFLD development. Thus, preventing $\mathrm{TG}$ and $\mathrm{CHO}$ accumulation in the liver may prove promising for NAFLD treatment.

Curcumin has been reported to alleviate HFD-induced obesity in mice through the inhibition of sterol regulatory element-binding proteins (SREBPs) such as SREBP-1 and SREBP-2. SREBPs are key transcription factors that modulate the expression of genes related to lipid synthesis (24). Specifically, SREBP-1c activation results in lipid-mediated lipotoxicity that contributes to metabolic syndromeassociated conditions including obesity, diabetes mellitus, hepato-steatosis, dyslipidemia, inflammation, and fibrosis in various organs $(24,25)$. SREBP-2 is a crucial transcription factor involved in the regulation of $\mathrm{CHO}$ metabolism
(26). We also confirmed that theracurmin treatment attenuated the increase in SREBP-1 induced by HFD (Supplementary Fig. 2). Curcumin has also been shown to reduce CHO accumulation via inhibition of 3-hydroxy-3-methylglutaryl-CoA (HMG-CoA) reductase, the rate-limiting enzyme of CHO synthesis, and acyl-CoA:cholesterol acyltransferase (ACAT), the main enzyme responsible for the intracellular esterification of CHO. It was also observed that theracurmin administration attenuated the increase HFD-associated increase in hepatic T-CHO levels (Fig. 2B). Although theracurmin treatment did not affect serum T-CHO in mice fed a HFD for 12 weeks, a low dosage of theracurmin $(200 \mathrm{mg} / \mathrm{kg}$ or $400 \mathrm{mg} / \mathrm{kg})$ resulted in a suppression of serum T-CHO levels in mice fed an HFD for 8 weeks (data not shown). Thus, it is plausible that theracurmin may regulate the extensive dysregulation of hepatic $\mathrm{CHO}$ homeostasis necessary for the development of hepatic steatosis found in HFD-fed mice. At the present time, the expression of hepatic HMG-CoA reductase and ACAT remains unexplored and will be a focus of further study.

Lipid accumulation as part of NAFLD progression results in increased vulnerability to oxidative stress, leading to an increase in inflammation, endoplasmic reticulum stress, mitochondrial dysfunction, and an inability of hepatocytes to synthesize endogenous antioxidants (27). Oxidative stress 
is caused by an imbalance between the formation of reactive nitrogen species and antioxidant defenses (28). Although the main processes for producing oxidizing species is related to the production of hydrogen peroxide in peroxisomes and oxidative metabolism in mitochondria, lipid peroxidation, the major consequence of oxidative stress, produces extremely reactive aldehyde components such as 4-hydroxy-2-nonenal and MDA, leading to intracellular damage in the liver $(29,30)$. Curcumin also has been shown to alleviate reactive oxygen species (ROS)-induced lipid peroxidation in mitochondria (31). These findings are consistent with our previous results, which saw theracurmin treatment contributing to a decrease in lipid peroxide levels and the induction of glutathione, both phenomena playing crucial roles in the detoxification and antioxidant systems involved in hepatic steatosis. Curcumin has been reported to induce the expression of antioxidant enzymes by upregulating nuclear factor erythroid-2-related factor-2 (Nrf2) $(32,33)$. In addition, curcumin contributed to a decrease in ROS production through the activation of Nrf2 in the muscles of HFD-fed mice (34). Due to this link between antioxidant enzyme expression and lipid peroxidation control, further studies should be conducted to address whether the decrease in lipid peroxidation observed after theracurmin treatment is related antioxidant enzyme levels or $\mathrm{Nrf} 2$ activation.

In conclusion, theracurmin appeared to play a crucial role in the prevention of hepatic steatosis by mediating the inhibition of TG/T-CHO biosynthesis and lipid peroxidation in the liver, suggesting that theracurmin can potentially be a new candidate as a therapeutic option for the treatment of fatty liver.

\section{ACKNOWLEDGMENTS}

This work was financially supported by Handok Inc.

\section{CONFLICT OF INTEREST}

The Authors who have taken part in this study declared that they do not have anything to disclose regarding funding or conflict of interest with respect to this manuscript. Hee Hye Yun is former employee of HANDOK, Inc.

Received December 26, 2018; Revised February 27, 2019; Accepted March 8, 2019

\section{REFERENCES}

1. Browning, J.D. and Horton, J.D. (2004) Molecular mediators of hepatic steatosis and liver injury. J. Clin. Invest., 114, 147-152.

2. Friedman, S.L., Neuschwander-Tetri, B.A., Rinella, M. and Sanyal, A.J. (2018) Mechanisms of NAFLD development and therapeutic strategies. Nat. Med., 24, 908-922.

3. Birkenfeld, A.L. and Shulman, G.I. (2014) Nonalcoholic fatty liver disease, hepatic insulin resistance, and type 2 diabetes. Hepatology, 59, 713-723.

4. Marra, F., Gastaldelli, A., Svegliati Baroni, G., Tell, G. and Tiribelli, C. (2008) Molecular basis and mechanisms of progression of non-alcoholic steatohepatitis. Trends Mol. Med., 14, 72-81.

5. Kotronen, A. and Yki-Jarvinen, H. (2008) Fatty liver: a novel component of the metabolic syndrome. Arterioscler. Thromb. Vasc. Biol., 28, 27-38.

6. Rinella, M.E. (2015) Nonalcoholic fatty liver disease: a systematic review. JAMA, 313, 2263-2273.

7. Sunagawa, Y., Hirano, S., Katanasaka, Y., Miyazaki, Y., Funamoto, M., Okamura, N., Hojo, Y., Suzuki, H., Doi, O., Yokoji, T., Morimoto, E., Takahashi, T., Ozawa, H., Imaizumi, A., Ueno, M., Kakeya, H., Shimatsu, A., Wada, H., Hasegawa, K. and Morimoto, T. (2015) Colloidal submicron-particle curcumin exhibits high absorption efficiency-a double-blind, 3-way crossover study. J. Nutr. Sci. Vitaminol., 61, 37-44.

8. Ohno, M., Nishida, A., Sugitani, Y., Nishino, K., Inatomi, O., Sugimoto, M., Kawahara, M. and Andoh, A. (2017) Nanoparticle curcumin ameliorates experimental colitis via modulation of gut microbiota and induction of regulatory $\mathrm{T}$ cells. PLOS ONE, 12, e0185999.

9. Farzaei, M.H., Zobeiri, M., Parvizi, F., El-Senduny, F.F., Marmouzi, I., Coy-Barrera, E., Naseri, R., Nabavi, S.M., Rahimi, R. and Abdollahi, M. (2018) Curcumin in liver diseases: a systematic review of the cellular mechanisms of oxidative stress and clinical perspective. Nutrients, 10, E855.

10. Maria, M., Eleni, P., George, V., Eftychia, T. and Constantinos, G. (2018) Effects of curcumin consumption on human chronic diseases: A narrative review of the most recent clinical data. Phytother. Res., 32, 957-975.

11. Imaizumi, A. (2015) Highly bioavailable curcumin (Theracurmin): its development and clinical application. PharmaNutrition, 3, 123-130.

12. Ding, L., Li, J., Song, B., Xiao, X., Zhang, B., Qi, M., Huang, W., Yang, L. and Wang, Z. (2016) Curcumin rescues high fat diet-induced obesity and insulin sensitivity in mice through regulating SREBP pathway. Toxicol. Appl. Pharmacol., 304, 99-109.

13. Shao, W., Yu, Z., Chiang, Y., Yang, Y., Chai, T., Foltz, W., Lu, H., Fantus, I.G. and Jin, T. (2012) Curcumin prevents high fat diet induced insulin resistance and obesity via attenuating lipogenesis in liver and inflammatory pathway in adipocytes. PLOS ONE, 7, e28784.

14. Sasaki, H., Sunagawa, Y., Takahashi, K., Imaizumi, A., Fukuda, H., Hashimoto, T., Wada, H., Katanasaka, Y., Kakeya, H., Fujita, M., Hasegawa, K. and Morimoto, T. (2011) Innovative preparation of curcumin for improved oral bioavailability. Biol. Pharm. Bull., 34, 660-665.

15. Cuomo, J., Appendino, G., Dern, A.S., Schneider, E., McKinnon, T.P., Brown, M.J., Togni, S. and Dixon, B.M. (2011) Comparative absorption of a standardized curcuminoid mixture and its lecithin formulation. J. Nat. Prod., 74, 664-669.

16. Gota, V.S., Maru, G.B., Soni, T.G., Gandhi, T.R., Kochar, N. and Agarwal, M.G. (2010) Safety and pharmacokinetics of a 
solid lipid curcumin particle formulation in osteosarcoma patients and healthy volunteers. J. Agric. Food Chem., 58, 2095-2099.

17. Sunagawa, Y., Wada, H., Suzuki, H., Sasaki, H., Imaizumi, A., Fukuda, H., Hashimoto, T., Katanasaka, Y., Shimatsu, A., Kimura, T., Kakeya, H., Fujita, M., Hasegawa, K. and Morimoto, T. (2012) A novel drug delivery system of oral curcumin markedly improves efficacy of treatment for heart failure after myocardial infarction in rats. Biol. Pharm. Bull., 35, 139-144.

18. Cichoz-Lach, H. and Michalak, A. (2014) Oxidative stress as a crucial factor in liver diseases. World J. Gastroenterol., 20, 8082-8091.

19. Oner-Iyidogan, Y., Kocak, H., Seyidhanoglu, M., Gurdol, F., Gulcubuk, A., Yildirim, F., Cevik, A. and Uysal, M. (2013) Curcumin prevents liver fat accumulation and serum fetuinA increase in rats fed a high-fat diet. J. Physiol. Biochem., 69, 677-686.

20. Liu, Y., Cheng, F., Luo, Y., Zhan, Z., Hu, P., Ren, H., Tang, H. and Peng, M. (2017) PEGylated curcumin derivative attenuates hepatic steatosis via CREB/PPAR-gamma/CD36 pathway. BioMed Res. Int., 2017, 8234507.

21. Greenberg, A.S., Coleman, R.A., Kraemer, F.B., McManaman, J.L., Obin, M.S., Puri, V., Yan, Q.W., Miyoshi, H. and Mashek, D.G. (2011) The role of lipid droplets in metabolic disease in rodents and humans. J. Clin. Invest., 121, 21022110.

22. Ioannou, G.N. (2016) The role of cholesterol in the pathogenesis of NASH. Trends Endocrinol. Metab., 27, 84-95.

23. Walenbergh, S.M. and Shiri-Sverdlov, R. (2015) Cholesterol is a significant risk factor for non-alcoholic steatohepatitis. Expert Rev. Gastroenterol. Hepatol., 9, 1343-1346.

24. Shimano, H. and Sato, R. (2017) SREBP-regulated lipid metabolism: convergent physiology - divergent pathophysiology. Nat. Rev. Endocrinol., 13, 710-730.

25. Yang, J.W., Kim, H.S., Im, J.H., Kim, J.W., Jun, D.W., Lim, S.C., Lee, K., Choi, J.M., Kim, S.K. and Kang, K.W. (2016) GPR119: a promising target for nonalcoholic fatty liver disease. FASEB J., 30, 324-335.
26. Moore, K.J., Rayner, K.J., Suarez, Y. and Fernandez-Hernando, C. (2011) The role of microRNAs in cholesterol efflux and hepatic lipid metabolism. Annu. Rev. Nutr., 31, 49-63.

27. Tariq, Z., Green, C.J. and Hodson, L. (2014) Are oxidative stress mechanisms the common denominator in the progression from hepatic steatosis towards non-alcoholic steatohepatitis (NASH)? Liver Int., 34, e180-e190.

28. Rolo, A.P., Teodoro, J.S. and Palmeira, C.M. (2012) Role of oxidative stress in the pathogenesis of nonalcoholic steatohepatitis. Free Radic. Biol. Med., 52, 59-69.

29. Spahis, S., Delvin, E., Borys, J.M. and Levy, E. (2017) oxidative stress as a critical factor in nonalcoholic fatty liver disease pathogenesis. Antioxid. Redox Signal., 26, 519-541.

30. Negre-Salvayre, A., Auge, N., Ayala, V., Basaga, H., Boada, J., Brenke, R., Chapple, S., Cohen, G., Feher, J., Grune, T., Lengyel, G., Mann, G.E., Pamplona, R., Poli, G., Portero-Otin, M., Riahi, Y., Salvayre, R., Sasson, S., Serrano, J., Shamni, O., Siems, W., Siow, R.C.M., Wiswedel, I., Zarkovic, K. and Zarkovic, N. (2010) Pathological aspects of lipid peroxidation. Free Radic. Res., 44, 1125-1171.

31. Wei, Q.Y., Chen, W.F., Zhou, B., Yang, L. and Liu, Z.L. (2006) Inhibition of lipid peroxidation and protein oxidation in rat liver mitochondria by curcumin and its analogues. Biochim. Biophys. Acta, 1760, 70-77.

32. Scapagnini, G., Vasto, S., Abraham, N.G., Caruso, C., Zella, D. and Fabio, G. (2011) Modulation of Nrf2/ARE pathway by food polyphenols: a nutritional neuroprotective strategy for cognitive and neurodegenerative disorders. Mol. Neurobiol., 44, 192-201.

33. Yang, C., Zhang, X., Fan, H. and Liu, Y. (2009) Curcumin upregulates transcription factor Nrf2, HO-1 expression and protects rat brains against focal ischemia. Brain Res., 1282, 133-141.

34. He, H.-J., Wang, G.-Y., Gao, Y., Ling, W.-H., Yu, Z.-W. and Jin, T.-R. (2012) Curcumin attenuates Nrf2 signaling defect, oxidative stress in muscle and glucose intolerance in high fat diet-fed mice. World J. Diabetes, 3, 94. 
(A)

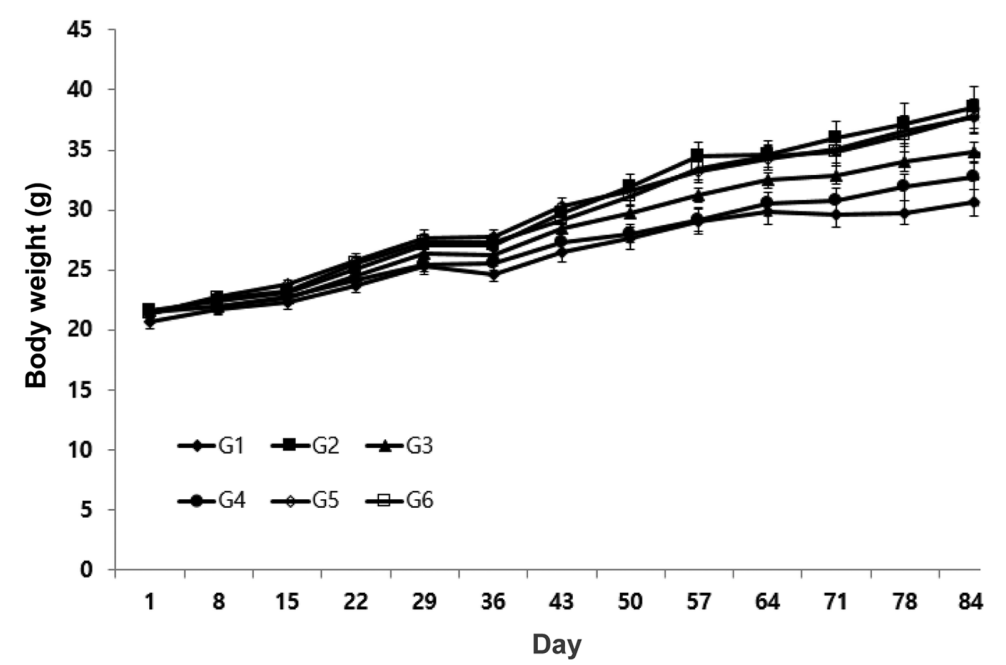

(B)

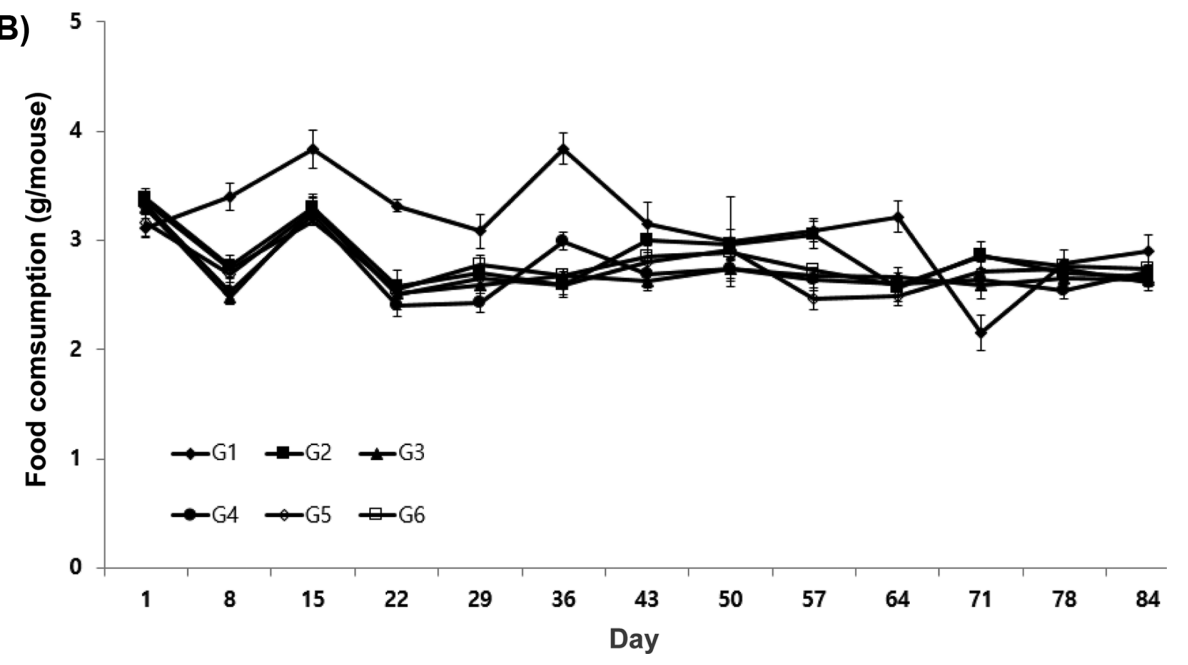

Supplementary Fig. 1. Effects of theracurmin on body weight gain and food intake in HFD-fed mice. Measurement of body weight gain and food consumptions. The mice were fed either a ND or HFD for 12 weeks. theracurmin (500, 1,000, and 2,000 mg/ $\mathrm{kg}$ ) or silymarin $(25 \mathrm{mg} / \mathrm{kg}$ ) (reference control) was administered to the mice at the same time. Data were expressed as mean $\pm \mathrm{SEM}$ statistically analyzed by LSD-test methods. Significant versus HFD-fed group, G1: ND (normal saline), $n=10 ; \mathrm{G2}$ : HFD + vehicle (normal saline), $n=10$; G3: HFD + theracurmin ( $500 \mathrm{mg} / \mathrm{kg} /$ day), $\mathrm{n}=10 ; \mathrm{G} 4: \mathrm{HFD}$ + theracurmin $(1,000 \mathrm{mg} / \mathrm{kg} /$ day), $\mathrm{n}=10 ; \mathrm{G} 5: \mathrm{HFD}$ + theracurmin (2,000 mg/kg/day), $\mathrm{n}=10 ; \mathrm{G} 6: \mathrm{HFD}$ + reference control (silymarin $25 \mathrm{mg} / \mathrm{kg} /$ day), $\mathrm{n}=10$. 

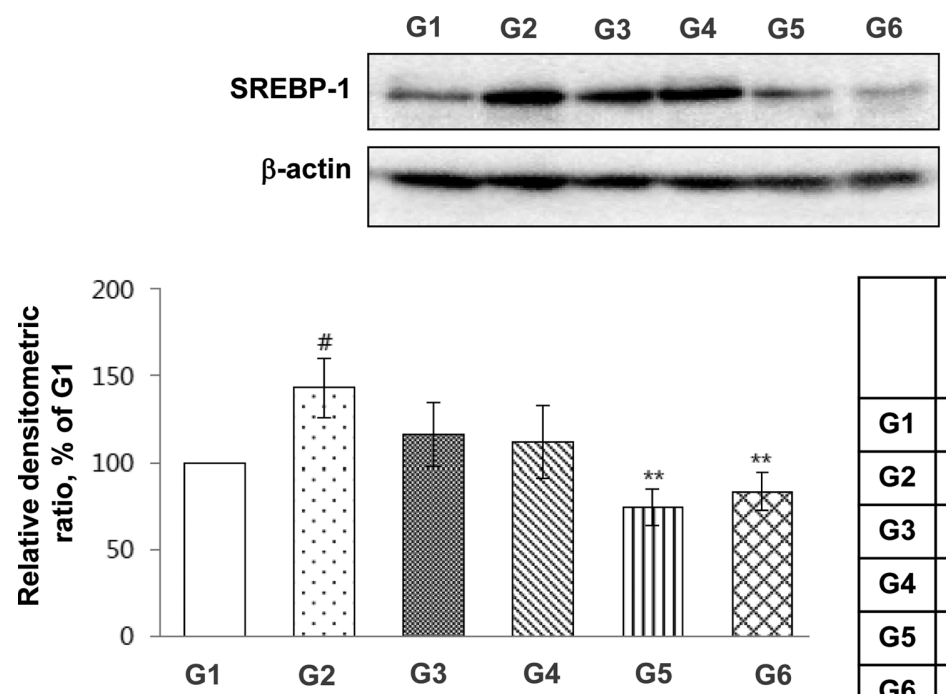

\begin{tabular}{|c|c|}
\hline & $\begin{array}{c}\text { Relative } \\
\text { densitometric } \\
\text { ratio, \% of G1 }\end{array}$ \\
\hline G1 & $100.00 \pm 0.00$ \\
\hline G2 & $143.25_{ \pm} 16.21^{\#}$ \\
\hline G3 & $116.41_{ \pm} \pm 17.44$ \\
\hline G4 & $111.86_{ \pm} 19.96$ \\
\hline G5 & $87.17_{ \pm} 15.14^{\star \star}$ \\
\hline G6 & $83.34_{ \pm} 10.54^{\star \star}$ \\
\hline
\end{tabular}

Supplementary Fig. 2. Effects of theracurmin on the hepatic expression of SREBP-1 in HFD-fed mice. Immunoblot analyses of SREBP-1. The mice were fed either a ND or HFD for 12 weeks. Theracurmin $(500,1,000$, and 2,000 mg/kg) or silymarin $(25 \mathrm{mg} / \mathrm{kg})$ (reference control) was administered to the mice at the same time. Data were expressed as mean \pm SEM statistically analyzed by LSD-test methods. G1: ND (normal saline), $\mathrm{n}=10 ; \mathrm{G} 2$ : HFD + vehicle (normal saline), $\mathrm{n}=10 ; \mathrm{G3}$ : HFD + theracurmin (500 mg/kg/day), $\mathrm{n}=10$; G4: HFD + theracurmin (1,000 mg/kg/day), $\mathrm{n}=10$; G5: HFD + theracurmin (2,000 mg/kg/day), $\mathrm{n}=10$; G6: HFD + reference control (silymarin $25 \mathrm{mg} / \mathrm{kg} /$ day), $\mathrm{n}=10$. 